\title{
ON THE INVERSE LIMIT OF EUCLIDEAN $N$-SPHERES $\left({ }^{1}\right)$
}

\author{
BY \\ MORTON BROWN
}

In [1] Bing constructed a 1-dimensional hereditarily indecomposable continuum which is the inverse limit of a sequence of circles $C_{i}$ and such that the maps $f_{i}: C_{i} \rightarrow C_{i-1}$ were of degree 1 . In this paper we show that the dimension cannot be raised, i.e., if $S=\operatorname{Lim}\left(S_{i}^{N}, f_{i}\right)$ where $S_{i}^{N}$ is an $N$-sphere and $f_{i}$ is essential, then $S$ is not hereditarily indecomposable. In doing so we further generalize Bing's definition of " $\epsilon$-crooked." Lemma 2 shows how to construct hereditarily indecomposable continua by making the bonding maps sufficiently crooked. In Lemma 3 we get a necessary crookedness condition on the bonding maps if the limit space is to be hereditarily indecomposable. The remainder of the paper is devoted to proving that in the case of $N$-spheres $(N>1)$ this condition cannot be satisfied.

Definitions and Notation. Let $X_{i}$ be a sequence of compact metric spaces, and for $i \geqq 2$ let $f_{i}$ be a map of $X_{i}$ into $X_{i-1}$. Then the subspace $\left({ }^{2}\right)\left({ }^{3}\right)$

$$
S=\left\{z \in \prod_{1}^{\infty} X_{i} \mid f_{i j}\left(z_{j}\right)=z_{i}\right\}
$$

of $\prod_{1}^{\infty} X_{i}$ is the inverse limit space of the inverse system $\left(X_{i}, f_{i}\right)$.

If $d_{i}$ is the diameter of $X_{i}$ then a metric for $\prod_{1}^{\infty} X_{i}$ (and hence for $S$ ) is given by $\left|z-z^{\prime}\right|=\sum_{1}^{\infty} 2^{-i} d_{i}^{-1}\left|z_{i}-z_{i}^{\prime}\right|$.

Let $f: X \rightarrow Y$ where $X, Y$ are metric spaces. Then for $\epsilon>0\left({ }^{4}\right)$,

$$
L(\epsilon, f)=\sup \left\{\delta \mid \begin{array}{c}
x, y \in X \text { and }|x-y|<\delta \\
\text { implies }|f(x)-f(y)|<\epsilon
\end{array}\right\} .
$$

If $X$ is compact then $L(\epsilon, f)>0$ for all $\epsilon>0$.

Let $f: X \rightarrow Y$ where $X$ is a topological space and $Y$ is a metric space. Let $\epsilon>0$. Then $f$ is $\epsilon$-crooked if for each path $g: I \rightarrow X\left(^{5}\right)$ there exist real numbers $t_{1}, t_{2}$ such that $0 \leqq t_{1} \leqq t_{2} \leqq 1$ and:

Presented to the Society April 25, 1959 under the title $A$ note on higher dimensional indecomposable continua; received by the editors September 25, 1959.

(1) This paper was written while the author was an Office of Naval Research Fellow at the University of Michigan.

(2) $f_{i j}=f_{i+1} f_{i+2} \cdots f_{j}, f_{i i}=1$.

(3) If $z \in \prod_{1}^{\infty} X_{i}$, then $z_{i}$ denotes the $i$ th coordinate of $z$. Hence $z=\left(z_{i}\right)$.

(4) $|x-y|$ denotes the distance from $x$ to $y$.

(5) $I$ denotes the unit interval [01]. 


$$
\begin{aligned}
& \left|f g(0)-f g\left(t_{2}\right)\right|<\epsilon, \\
& \left|f g\left(t_{1}\right)-f g(1)\right|<\epsilon .
\end{aligned}
$$

A metric space $Y$ is $\epsilon$-crooked if the identity map 1: $Y \rightarrow Y$ is $\epsilon$-crooked $\left({ }^{6}\right)$.

A continuum $M$ is indecomposable if it is not the union of two proper subcontinua. If every subcontinuum of $M$ is indecomposable then $M$ is hereditarily indecomposable.

Lemma 1. Let $K$ be a metric space. Suppose $K_{1}, K_{2}, \ldots$ is a sequence of Peanian continua in $K$ such that $K_{1} \supset K_{2} \supset \ldots$. Then $\bigcap_{1}^{\infty} K_{n}$ is hereditarily indecomposable if and only if there is a null sequence $\left(\epsilon_{n}\right)$ of positive real numbers such that $K_{n}$ is $\epsilon_{n}$-crooked.

Proof of sufficiency. Suppose $M$ is a subcontinuum of $\bigcap_{1}^{\infty} K_{n}$ and $M=A \cup B$, where $A, B$ are proper subcontinua of $M$. Let $a \in A-B, b \in B-A$. Then there is an $n$ such that $\epsilon_{n}<\min (|a-B|,|b-A|)\left({ }^{7}\right)$. Let $O_{A}, O_{B}$ be connected open subsets of $K_{n}$ such that $A \subset O_{A}, B \subset O_{B},\left|a-\bar{O}_{B}\right|>\epsilon_{n}$, and $\left|b-\bar{O}_{A}\right|>\epsilon_{n}$. Let $x \in O_{A} \cap O_{B}$. Then there are paths $\alpha, \beta: I \rightarrow K_{n}$ such that $\alpha(0)=a, \alpha(1)=x, \beta(0)=x, \beta(1)=b, \alpha(I) \subset O_{A}$, and $\beta(I) \subset O_{B}$. Let $g: I \rightarrow K$ where

$$
g(t)=\left\{\begin{array}{lr}
\alpha(2 t), & 0 \leqq t \leqq 1 / 2, \\
\beta(2 t-1), & 1 / 2 \leqq t \leqq 1
\end{array}\right.
$$

Since $K_{n}$ is $\epsilon_{n}$-crooked there exist $t_{1}, t_{2}$ such that $t_{1} \leqq t_{2},\left|a-g\left(t_{2}\right)\right|<\epsilon_{n}$ and $\left|g\left(t_{1}\right)-b\right|<\epsilon_{n}$. Hence $t_{2}<1 / 2$ and $t_{1}>1 / 2$. But this contradicts the fact that $t_{1} \leqq t_{2}$.

Proof of necessity. Suppose $\bigcap_{1}^{\infty} K_{n}$ is hereditarily indecomposable and for some $\epsilon>0$ there is a sequence $n_{i}$ such that $K_{n_{i}}$ is not $\epsilon$-crooked. Then for each $i$ there is a path $\alpha_{i}: I \rightarrow K_{n_{i}}$, and real numbers $t_{i}$ such that $\left|\alpha_{i}\left(\left[0 t_{i}\right]\right)-\alpha_{i}(1)\right|$ $\geqq \epsilon$ and $\left|\alpha_{i}\left(\left[t_{i} 1\right]\right)-\alpha_{i}(0)\right| \geqq \epsilon\left({ }^{8}\right)$. Let $A_{n_{i}}=\alpha_{i}\left(\left[0 t_{i}\right]\right), B_{n_{i}}=\alpha_{i}\left(\left[t_{i}, 1\right]\right), M_{n_{i}}$ $=\alpha_{i}(I)$. Then $\lim \sup A_{n_{i}}, \lim \sup B_{n_{i}}$ are proper subcontinua of the continuum $\lim \sup M_{n_{i}}$, and $\lim \sup M_{n_{i}}=\lim \sup A_{n_{i}} \cup \lim \sup B_{n_{i}}$.

Lemma 2. Let $S=\lim \left(X_{i}, f_{i}\right)$ where the $X_{i}$ are Peanian continua with diameters $d_{i}$. Suppose for all $n, f_{n}$ is $\epsilon_{n}$-crooked where $\epsilon_{n}<\min _{i<n-1} L\left(2^{-n} d_{i}, f_{i_{n-1}}\right)$. Then $S$ is hereditarily indecomposable.

Proof. Let $K_{n}=\left\{z \in \prod_{1}^{\infty} X_{i} \mid f_{i j}\left(z_{j}\right)=z_{i}\right.$ for $\left.i<j \leqq n\right\}$. Then $K_{n}$ is homeomorphic to $X_{n}$ and $S=\bigcap_{n-1}^{\infty} K_{n}$. Now $K_{n}$ is $2^{3-n}$-crooked. For if $g: I \rightarrow K_{n}$ is a path in $K_{n}$, then $\left({ }^{9}\right)$

(8) It may be noted that both definitions of $\epsilon$-crooked (for maps and for spaces) depend on the given metric.

(7) $|x-Y|$ denotes the distance from $x$ to $Y$.

(8) We may assume without loss of generality that $\alpha_{i}\left(t_{i}\right)$ converges.

$(\vartheta) \pi_{n}$ denotes the map collapsing each point of $\prod_{1}^{\infty} X_{i}$ onto its $n$th coordinate. 


$$
I \stackrel{g}{\longrightarrow} K_{n} \stackrel{\pi_{n}}{\longrightarrow} X_{n} \stackrel{f_{n}}{\longrightarrow} X_{n-1} \stackrel{f_{i n-1}}{\longrightarrow} X_{i} .
$$

Since $f_{n}$ is $\boldsymbol{\epsilon}_{n}$-crooked, there exist real numbers $t_{1}, t_{2}$ such that $0 \leqq t_{1} \leqq t_{2} \leqq 1$, $\left|f_{n} \pi_{n} g(0)-f_{n} \pi_{n} g\left(t_{2}\right)\right|<\epsilon_{n}$, and $\left|f_{n} \pi_{n} g\left(t_{1}\right)-f_{n} \pi_{n} g(1)\right|<\epsilon_{n}$. Let $\alpha=g(0), \beta=g\left(t_{2}\right)$ $\cdot\left(\alpha, \beta \in K_{n}\right)$. Then $\left|f_{n} \pi_{n}(\alpha)-f_{n} \pi_{n}(\beta)\right|<\epsilon_{n}$, i.e. $\left|\alpha_{n-1}-\beta_{n-1}\right|<\epsilon_{n}$. Since $\epsilon_{n}<L\left(2^{-n} d_{i}, f_{i n-1}\right),\left|\alpha_{i}-\beta_{i}\right|<2^{-n} d_{i}$ for $i=1,2, \cdots,(n-2)$. Hence

$$
\begin{aligned}
|\alpha-\beta|=\sum_{1}^{\infty} 2^{-i} d_{i}^{-1}\left|\alpha_{i}-\beta_{i}\right| & \leqq \sum_{1}^{n-2} 2^{-i} d_{i}^{-1}\left|\alpha_{i}-\beta_{i}\right|+2^{2-n} \\
& \leqq \sum_{1}^{n-2} 2^{-i} d_{i}^{-1} 2^{-n} d_{i}+2^{2-n} \\
& \leqq 2^{-n} \sum_{1}^{n-2} 2^{-i}+2^{2-n} \\
& <2^{-n}+2^{2-n} \\
& <2^{3-n} .
\end{aligned}
$$

Hence $\left|g(0)-g\left(t_{2}\right)\right|<2^{3-n}$. Similarly, $\left|g\left(t_{1}\right)-g(1)\right|<2^{3-n}$. Hence $K_{n}$ is $2^{3-n_{-}}$ crooked. But $S=\cap_{n=1}^{\infty} K_{n}$, so by Lemma $1, S$ is hereditarily indecomposable.

Lemma 3. Let $S=\lim \left(X_{i}, f_{i}\right)$ where the $X_{i}$ are Peanian continua and $S$ is hereditarily indecomposable. Then for any $\epsilon>0$ there is an $n$ such that $f_{1 n}$ is $\boldsymbol{\epsilon}$-crooked.

Proof. By Lemma 1 there is an $n$ such that $K_{n}$ is $2^{-1} \epsilon d_{1}^{-1}$-crooked. Let $g: I \rightarrow X_{n}$ be a path in $X_{n}$. Let $x_{i} \in X_{i}$ for $i=n+1, n+2, \cdots$. Let $\bar{g}: I \rightarrow K_{n}$ by

$$
\bar{g}(t)=\left(f_{1 n} g(t), f_{2 n} g(t), \cdots, g(t), x_{n+1}, x_{n+2}, \cdots\right) .
$$

Since $K_{n}$ is $2^{-1} \epsilon d_{1}^{-1}$-crooked there exist $t_{1} \leqq t_{2}$ such that $\left|\bar{g}(0)-\bar{g}\left(t_{2}\right)\right|<2^{-1} d_{1}^{-1} \epsilon$, $\left|\bar{g}\left(t_{1}\right)-\bar{g}(1)\right| 2^{-1} d^{-1} \epsilon$. Now $\left|\bar{g}(0)-\bar{g}\left(t_{2}\right)\right|=\sum_{i=1}^{n} 2^{-i} d_{i}^{-1}\left|f_{\text {in }} g(0)-f_{\text {in }} g\left(t_{2}\right)\right|$. Hence $\sum_{i=1}^{n} 2^{-i} d_{i}^{-1}\left|f_{i n} g(0)-f_{\text {in }} g\left(t_{2}\right)\right|<2^{-1} d_{1}^{-1} \epsilon$. In particular

$$
2^{-1} d_{1}^{-1}\left|f_{1 n} g(0)-f_{1 n} g\left(t_{2}\right)\right|<2^{-1} d_{1}^{-1} \epsilon \text {, or }\left|f_{1 n} g(0)-f_{1 n} g\left(t_{2}\right)\right|<\epsilon .
$$

Similarly $\left|f_{\text {in }} g\left(t_{1}\right)-f_{\text {in }} g(1)\right|<\epsilon$. Hence $f_{1 n}$ is $\epsilon$-crooked.

Lemma 4. Let $Y$ be a metric space, $X$ a topological space, and $f, g$ two maps of $X$ into $Y$ such that $\|f-g\|<\delta$. Then if $f$ is $\epsilon$-crooked, $g$ is $(\epsilon+2 \delta)$-crooked.

Proof. Suppose $\alpha: I \rightarrow X$ is a path in $X$. Since $f$ is $\epsilon$-crooked there exist $t_{1} \leqq t_{2}$ such that $\left|f \alpha(0)-f \alpha\left(t_{2}\right)\right|<\epsilon,\left|f \alpha\left(t_{1}\right)-f \alpha(1)\right|<\epsilon$. Hence

$$
\begin{aligned}
\left|g \alpha(0)-g \alpha\left(t_{2}\right)\right| & \leqq|g \alpha(0)-f \alpha(0)|+\left|f \alpha(0)-f \alpha\left(t_{2}\right)\right|+\left|f \alpha\left(t_{2}\right)-g \alpha\left(t_{2}\right)\right| \\
& <\|g-f\|+\epsilon+\|g-f\| \\
& <[\epsilon+2 \delta .
\end{aligned}
$$


Similarly $\left|g \alpha\left(t_{1}\right)-g \alpha(1)\right|<\epsilon+2 \delta$.

Let $T$ be a triangulation of the $N$-sphere $S^{N}(N>1)$ and suppose $\bar{f}: T^{n} \rightarrow T^{m}$ is a chain map of the $n$th barycentric subdivision of $T$ onto the $m$ th barycentric subdivision of $T$. Let $f:\left|T^{n}\right| \rightarrow\left|T^{m}\right|$ be the induced map of $S^{N}$ upon itself and suppose $f$ is essential. Finally let $\sigma$ be a $N$-simplex of $T$. An open subset $o$ of $S^{N}$ is said to be inessential $\bmod |\sigma|$ if there is a homotopy $F: S^{N}$ $X I \rightarrow S^{N}$ such that

(a) $F_{0}=f$,

(b) $F_{t} \mid\left(S^{N}-o\right)=f$,

(c) $F_{1}(o) \subset|\sigma|$.

Otherwise $o$ is essential mod $|\sigma|$.

Lemma 5. Let o be essential mod $|\sigma|$. Then if $F$ is any homotopy of $S^{N} \times I$ into $S^{N}$ satisfying the above conditions (a) and (b), $F_{1}(o) \supset S^{N}-|\sigma|$.

Proof. Suppose $x_{0} \in S^{N}-\left[|\sigma| \cup F_{1}(o)\right]$. Since $|\sigma|$ is a strong deformation retract of $S^{N}-x_{0}$, there is a homotopy $G:\left(S^{N}-x_{0}\right) \times I \rightarrow S^{N}$ such that $G_{0}=1$, $G_{t}|| \sigma \mid=1$, and $G_{1}\left(S^{N}-x_{0}\right) \subset|\sigma|$. Let $H: S^{N} \times I \rightarrow S^{N}$ by:

$$
H(x, t)=\left\{\begin{array}{lr}
F(x, 2 t), & 0 \leqq t \leqq 1 / 2, \\
f(x), & 1 / 2 \leqq t \leqq 1, x \notin o, \\
G\left(F_{1}(x), 2 t-1\right), & 1 / 2 \leqq t \leqq 1, x \in o .
\end{array}\right.
$$

Then $H_{0}=f, H_{t} \mid\left(S^{N}-o\right)=f$, and $H_{1}(o)=G_{1}\left(F_{1}(o)\right) \subset G_{1}\left(S^{N}\right) \subset|\sigma|$. But this contradicts the assumption that $o$ is essential mod $|\sigma|$.

Lemma 6. Suppose $o_{1}, o_{2}$ are disjoint open subsets of $S^{N}$. Then if each is inessential mod $|\sigma|$ so is their union.

Proof. Let $F, G$ be the homotopies corresponding to $o_{1}, o_{2}$ respectively. Let $H: S^{N} \times I \rightarrow S^{N}$ by:

$$
H(x, t)=\left\{\begin{array}{lc}
F(x, 2 t), & 0 \leqq t \leqq 1 / 2, \\
G(x, 2 t-1), & 1 / 2 \leqq t \leqq 1, x \in o_{1}, \\
F(x, 1), & 1 / 2 \leqq t \leqq 1, x \in o_{1} .
\end{array}\right.
$$

Lemma 7. If $X$ is a component of $f^{-1}(|\sigma|)$, then at least one complementary domain of $X$ is essential mod $|\sigma|$.

Proof. Let $o_{1}, o_{2}, \cdots, o_{r}$ be the complementary domains of $X$. If each $o_{i}$ is inessential then by Lemma 6 so is their union $S^{N}-X$. Hence there is a homotopy $F: S^{N} \times I \rightarrow S^{N}$ such that $F_{0}=f, F_{t} \mid X=f$, and $F_{1}\left(S^{N}-X\right) \subset|\sigma|$. But then $F_{1}\left(S^{N}\right) \subset|\sigma|$. Hence $F_{1}$ is inessential. But $F_{1}$ is homotopic to $f$ and $f$ is essential.

Lemma 8. Suppose $\sigma_{1}, \sigma_{2}, \sigma_{3}$ are three $N$-simplexes of $T$ whose geometric realizations are pairwise disjoint. Then there is an integer $i_{0}\left(i_{0}=1,2,3\right), a$ component $X$ of $f^{-1}\left(\left|\sigma_{i_{0}}\right|\right)$, and a complementary domain of $X$ such that: 
(a) $o$ is essential mod $\left|\sigma_{i_{0}}\right|$.

(b) If $Y$ is any component of $f^{-1}\left(\left|\sigma_{i}\right|\right)(i=1,2,3)$ in o and $o^{\prime}$ is a complementary domain of $Y$ lying in $o$, then $o^{\prime}$ is inessential $\bmod \left|\sigma_{i}\right|$.

Proof. Let $X_{1}$ be a component of $f^{-1}\left(\left|\sigma_{1}\right|\right)$. By Lemma $7, X_{1}$ has a complementary domain $o_{1}$ which is essential $\bmod \left|\sigma_{1}\right|$. If the pair $\left(X_{1}, o_{1}\right)$ does not satisfy condition (b) there is an integer $i_{2}\left(i_{2}=1,2,3\right)$, a component $X_{2}$ of $f^{-1}\left(\left|\sigma_{i_{2}}\right|\right)$, and a complementary domain $o_{2}$ of $X_{2}$ such that $o_{2} \subset o_{1}$ and $o_{2}$ is essential mod $\left|\sigma_{2}\right|$. Continuing in this fashion we must arrive at the required pair $(X, o)$ after a finite number of steps.

LEMmA 9. Let $\sigma_{1}, \sigma_{2}, \sigma_{3}$ be three $N$-simplexes of $T$ whose geometric realizations are pairwise disjoint. Let $p_{1}, p_{2}, p_{3}$ be interior points of $\left|\sigma_{1}\right|,\left|\sigma_{2}\right|,\left|\sigma_{3}\right|$ respectively. Then for some permutation $(i, j, k)$ of $(1,2,3)$ there is a component $X$ of $f^{-1}\left(\left|\sigma_{i}\right|\right)$, a complementary domain o of $X$, and points $p_{j}^{\prime}, p_{k}^{\prime}$ of $o$ such that:

(a) $f\left(p_{j}^{\prime}\right)=p_{j}$,

(b) $f\left(p_{k}^{\prime}\right)=p_{k}$,

(c) $f^{-1}\left(\left|\sigma_{j}\right|\right)$ does not separate $p_{k}^{\prime}$ from $X$,

(d) $f^{-1}\left(\left|\sigma_{k}\right|\right)$ does not separate $p_{j}^{\prime}$ from $X$.

Proof. Let $i, o, X$ be the $i_{0}, o, X$ of Lemma 8 . Let $j, k$ be any permutation of the remaining integers. Let $Q_{1}, Q_{2}, \cdots, Q_{w}$ be those components of $f^{-1}\left(\left|\sigma_{j}\right|\right)$ in $o$ which are not separated from $X$ by any other component of $f^{-1}\left(\left|\sigma_{j}\right|\right)$. For $1 \leqq t \leqq w$ let $W_{t}$ be the union of the complementary domains of $Q_{t}$ not containing $X$. Then the $W_{t}$ are pairwise disjoint. From condition (b) of Lemma 8 , each component of $W_{t}$ is inessential mod $\left|\sigma_{j}\right|$. Hence by Lemma 6 , each $W_{t}$ is inessential mod $\left|\sigma_{j}\right|$. Again, by Lemma $6, U_{1}^{w} W_{t}$ is inessential $\bmod \left|\sigma_{j}\right|$. Hence there is a homotopy $F: S^{N} \times I \rightarrow S^{N}$ such that $F_{0}=f$, $F_{t} \mid\left(S^{N}-\bigcup_{1}^{w} W_{t}\right)=f$, and $F_{1}\left(\bigcup_{1}^{w} W_{t}\right) \subset\left|\sigma_{j}\right|$. By Lemma 5, since $o$ is essential $\bmod \left|\sigma_{i}\right|$, there is a point $p_{k}^{\prime} \in o$ such that $F_{1}\left(p_{k}^{\prime}\right)=p_{k}$. Since $F_{1}\left(\cup_{1}^{w} W_{t}\right)$ $C\left|\sigma_{j}\right|, p_{k}^{\prime} \notin \cup_{1}^{w} W_{t}$. Hence no $Q_{t}$ (and therefore no component of $f^{-1}\left(\left|\sigma_{j}\right|\right.$ ) separates $p_{k}^{\prime}$ from $X$. It follows from the unicoherence $\left({ }^{10}\right)$ of $S^{N}$ that $f^{-1}\left(\left|\sigma_{j}\right|\right)$ does not separate $p_{k}^{\prime}$ from $X$. In the same manner we can find a $p_{j}^{\prime} \in o \cap f^{-1}\left(p_{j}\right)$ such that $f^{-1}\left(\left|\sigma_{k}\right|\right)$ does not separate $p_{j}^{\prime}$ from $X$.

Lemma 10. Let $T$ be a triangulation of $S^{N}(N>1)$ and $\bar{f}: T^{n} \rightarrow T^{m}$ a chain map such that the induced map $f:\left|T^{n}\right| \rightarrow\left|T^{m}\right|$ is essential. Suppose $\sigma_{1}, \sigma_{2}, \sigma_{3}$ are $N$-simplexes of $T$ whose geometric realizations are pairwise disjoint. Let $p_{1}, p_{2}, p_{3}$ be points interior to $\left|\sigma_{1}\right|,\left|\sigma_{2}\right|,\left|\sigma_{3}\right|$ respectively. Finally, suppose $\epsilon<\min _{i} D\left(p_{i}, S^{N}-\left|\sigma_{i}\right|\right)$. Then $f$ is not $\epsilon$-crooked.

Proof. The hypotheses of this lemma include those of Lemma 9. Rather than restating the conclusions of Lemma 9 let us bodily incorporate its con-

(10) It is here that the proof breaks down for $N=1$. 
clusions and notation. It follows from conditions (c) and (d) and from the unicoherence of $S^{N}$ that there exist arcs $\left[p_{j}^{\prime} x_{1}\right]$ and $\left[p_{k}^{\prime} x_{2}\right]$ in $S^{N}$ such that $x_{1} \cup x_{2} \subset X, \quad\left[p_{j}^{\prime} x_{1}\right] \cap f^{-1}\left(\left|\sigma_{k}\right|\right)=\left[p_{k}^{\prime} x_{2}\right] \cap f^{-1}\left(\left|\sigma_{j}\right|\right)=0, \quad\left[p_{k}^{\prime} x_{2}\right] \cap X=x_{2}$, and $\left[p_{j}^{\prime} x_{1}\right] \cap X=x_{1}$. Since $X$ is a connected polyhedron there is an arc $\left[x_{1} x_{2}\right]$ in $X$. Let $h_{1}, h_{2}, h_{3}$ be homeomorphisms of $I$ into $\left[p_{j}^{\prime} x_{1}\right],\left[x_{1} x_{2}\right]$, and $\left[x_{2} p_{k}^{\prime}\right]$ respectively, such that $h_{1}(0)=p_{j}^{\prime}, h_{2}(0)=x_{1}, h_{3}(0)=x_{2}$. Let $g: I \rightarrow S^{N}$ be defined by:

$$
g(t)=\left\{\begin{array}{lr}
h_{1}(3 t), & 0 \leqq t \leqq 1 / 3, \\
h_{2}(3 t-1), & 1 / 3 \leqq t \leqq 2 / 3, \\
h_{3}(3 t-2), & 2 / 3 \leqq t \leqq 1 .
\end{array}\right.
$$

If $f$ were $\epsilon$-crooked there would be real numbers $t_{1}, t_{2}$ such that $t_{1} \leqq t_{2}$, $\left|f g(0)-f g\left(t_{2}\right)\right|<\epsilon$, and $\left|f g\left(t_{1}\right)-f g(1)\right|<\epsilon$. Now $f g(0)=p_{j}$ and $f g(1)=p_{k}$.

Since $\left(\left[p_{j}^{\prime} x_{1}\right] \cup\left[x_{1} x_{2}\right]\right) \cap f^{-1}\left(\left|\sigma_{k}\right|\right)=0,\left|f g(t)-p_{k}\right|>\epsilon$ for $0 \leqq t \leqq 2 / 3$. Hence $t_{1}>2 / 3$. Similarly $\left|f g(t)-p_{j}\right|>\epsilon$ for $1 / 3 \leqq t \leqq 1$. Hence $t_{2}<1 / 3$. But this contradicts the assumption that $t_{1} \leqq t_{2}$. Hence $f$ is not $\epsilon$-crooked.

Theorem 1. Let $N>1$. Then there is an $\epsilon>0$ such that no essential map of $S^{N}$ upon itself is $\epsilon$-crooked.

Proof. Let $T$ be a triangulation of $S^{N}$ fine enough to insure the existence of three $N$-simplexes $\sigma_{1}, \sigma_{2}, \sigma_{3}$ whose geometric realizations are pairwise disjoint. Let $p_{i}$ be an interior point of $\left|\sigma_{i}\right|(i=1,2,3)$ and let

$$
2 \epsilon<\min _{i} D\left(p_{i}, S^{N}-\left|\sigma_{i}\right|\right) .
$$

Suppose now that $g$ is any essential map of $S^{N}$ upon itself. By the Simplicial Approximation Theorem there are barycentric subdivisions $T^{n}, T^{m}$ of $T$ and a chain map $\bar{f}: T^{n} \rightarrow T^{m}$ such that if $f$ is the map of $\left|T^{n}\right| \rightarrow\left|T^{m}\right|$ induced by $\bar{f}$, then $f$ is homotopic to $g$ and $\|f-g\|<\epsilon / 2$. Suppose $g$ is $\epsilon$-crooked. Then by Lemma $4 f$ is $2 \epsilon$-crooked. But by Lemma $10 f$ cannot be $2 \epsilon$-crooked. Hence $g$ cannot be $\epsilon$-crooked.

THEOREM 2. Let $X=\lim \left(X_{i}, f_{i}\right)$ where for some $N>1$ each $X_{i}$ is an $N$ sphere and $f_{i}$ is essential. Then $X$ is not hereditarily indecomposable.

Proof. This theorem is a direct consequence of Theorem 1 and Lemma 3.

\section{REFERENCES}

1. R. H. Bing, Concerning hereditarily indecomposable continua, Pacific J. Math. vol. 1 (1951) pp. 43-51.

2. Higher dimensional hereditarily indecomposable continua, Trans. Amer. Math. Soc. vol. 71 (1951) pp. 267-273.

3. H. Freudenthal, Entwicklungen von Raumen und ihrer Gruppen, Compositio Math. vol. 4 (1937) pp. 145-234.

UNIVERSITY OF MichIGAN, ANN ARBor, MichigaN 